\title{
A contribution to the theory of fertility and
}

\section{economic development}

\author{
Luca Gori*• Mauro Sodini ${ }^{\dagger}$
}

June 3, 2019

${ }^{*}$ L. Gori (corresponding author), Department of Law, University of Pisa, Via Collegio Ricci, 10, I-56126 Pisa (PI), Italy, e-mail: luca.gori@unipi.it or dr.luca.gori@gmail.com., tel.: +390502212 847.

${ }^{\dagger}$ M. Sodini, Department of Economics and Management, University of Pisa, Via Cosimo Ridolfi, 10, I-56124 Pisa (PI), Italy, e-mail: mauro.sodini@unipi.it, tel.: +3905022 16 234, fax: +3905022 10603. 


\section{Fertility and economic development}

Corresponding author: Luca Gori, Department of Law, University of Pisa, Via Collegio Ricci, 10, I-56126 Pisa (PI), Italy, e-mail: luca.gori@unipi.it or dr.luca.gori@gmail.com., tel.: $+390502212847$. 
Abstract The aim of this research is to build on a theory for explaining economic development in a (neoclassical) growth model with endogenous fertility. The economy is inhabited by overlapping generations of rational and identical individuals and identical competitive firms producing with a constant-returns-to-scale technology and no externalities. From a theoretical perspective, the distinguishing feature of this work is that endogenous fertility per se explains the existence of low and high development regimes. It provides different reasons (history-driven or expectation-driven) why some countries enter development trajectories with high GDP and low fertility and others experience under performances with low GDP and high fertility. The model is also capable to reproduce fertility fluctuations and explain the baby busts and baby booms observed in the last century in some developed countries.

Keywords Economic development; Endogenous fertility; Local and global indeterminacy; OLG model

JEL Classification $\quad$ C61; C62; J1, J22; O41 


\section{Introduction}

"...if the theory is complicated, it's wrong." Richard P. Feynman

The present research aims at explaining the reasons why some countries develop, and others remain entrapped in stagnation or poverty by using a neoclassical growth model with overlapping generations (OLG) and endogenous fertility (child quantity). The work introduces a novel utility-driven mechanism describing the existence of different convergence groups of countries and confirms the empirical findings of Wang et al. (1994), Palivos (1995) and the related literature on fertility and growth. It also provides a new theoretical reason why economies starting from similar initial conditions experience different development trajectories or initially poorer (resp. richer) economies enter a phase of sustained development (resp. under-development) with large values of GDP and low fertility rates (resp. small values of GDP and high fertility rates), as several European, Latin American and East Asian countries show starting from the end of World War II. From a theoretical perspective, this work complements the continuous-time optimal growth model with child quantity and child quality of Palivos (1995), where multiple steady states and problems of coordination failures were production-driven. The article now proceeds by discussing the main motivations and the links to the related literature.

Human beings in Western countries have experienced tremendous improvements in both the standard of living and quality of life in the past two centuries, though there is still no clear consensus on which the main sources of this development were. However, demographic variables were recognised to play a preeminent role as determinants of long-term macroeconomic outcomes of nations, and the interaction between demographics and economics has become the object of a growing body of theoretical and empirical studies [e.g., Fogel (2004); Galor 
(2011); Cervellati and Sunde (2013)]. The influence of longevity and fertility on economic growth (a concept referred to the growth of an economic variable such as GDP per capita) and development (a multi-dimensional phenomenon related - amongst other things - to fertility, life expectancy, poverty, quality of institutions, the distribution of income and so on) has led several economists to consider them as endogenous variables and tackle this issue in models that - since the pioneering work of Becker (1960) - have originated the Unified Growth Theory (UGT) [Galor and Weil (2000); Galor and Moav (2002, 2004); Galor (2011)]. This theory aims at explaining the process of economic development based on the interaction between endogenous technological progress and human capital formation by showing that endogenous fertility (quantity-quality trade-off) [Galor and Weil (2000)] and endogenous human evolution [Galor and Moav (2002)] are relevant causes of the demographic transition, i.e. the transition from stagnation to growth is accompanied by a demographic shift from high to low birth and death rates [Jones and Tertilt (2006)]. A distinctive feature of this literature is the building of a conceptual framework capable of describing the process of development across nations and continents. This process is usually divided in three phases: 1) Malthusian epoch (a long period that ends up almost at 1750 AD). 2) Post-Malthusian regime (1750-1870). 3) Modern growth regime (1870-today). The works belonging to this literature have substantially modified the standard OLG model by including some additional ingredients, such as human capital accumulation, child and adult mortality and so on. Except for some seminal contributions that tend to emphasise the importance of fertility and child mortality as determinants of the economic and demographic transition [Galor and Weil (2000); Doepke (2005)], the mechanisms leading from a phase of pre-industrialisation to a phase of intensive industrial production are usually technology-and-mortality-driven [Cervellati and Sunde (2005, 2015); Fiaschi and Fioroni (2014)]. In these works, fertility can explain the 
phases of the demographic transition, but it does not represent the triggering factor amongst the various stages of development.

There are several contributions analysing problems of economic development in growth models with endogenous fertility that do not strictly belong to the UGT. These works aim at explaining the reasons why some countries achieve high values of GDP and low fertility rates and others remain entrapped in a situation where GDP is low, and fertility is high. In most cases, scholars have used the OLG framework. This is because it represents a natural basis where including demographic variables, although there are some works framed in a continuoustime set up with infinite horizon optimising agents [Wang et al. (1994); Palivos (1995); Palivos et al. (1997)]. Within the class of OLG models with finite lived individuals, we mention here the articles of Galor and Weil (1996), Blackburn and Cipriani (2002) and Fanti and Gori (2014) that come to light some distinct reasons why multiple stationary equilibria exist in models that substantially modified the basic OLG framework. The seminal work of Galor and Weil (1996) explained the fertility transition by including gender differences. The decrease in fertility and the increase in output growth were due to a threefold reason: the increase in capital per worker and women's relative wages; the resulting increase in the opportunity cost of children; the additional increase in capital per worker due to the fertility decline. Multiple development regimes were possible because of the positive effect on the rate of output growth caused by women joining the labour force. Differently, Blackburn and Cipriani (2002) and Fanti and Gori (2014) built on growth models where changes in adult mortality - driven, respectively, by education and public health expenditure - are responsible for the existence of different longterm outcomes. The main finding is that poor (resp. rich) countries tend to have high (resp. low) fertility and mortality rates as well as low (resp. high) GDP. These models can explain 
the demographic transition, but endogenous fertility choices do not represent the main reasons why countries develop.

In line with Galor and Weil (1996), this article explains the fertility transition within the wider phenomenon of the demographic transition without resorting to the paradigm of the child quantity-quality trade-off. This explanation can be history driven or expectations driven, so that underdevelopment may be a problem of fertility-driven coordination failures: agents know that there are multiple equilibria but do not know how to avoid Pareto dominated outcomes. ${ }^{1}$ The model is capable of describing the coexistence of a high regime of development (the accumulation of capital is high, and fertility is low), which resembles the Modern growth regime, and a low regime of development (the accumulation of capital is low, and fertility is high), which resembles the Malthusian regime. We pinpoint that the main results were obtained in a standard framework with homogeneous agents and no externalities in production.

The rest of the article proceeds as follows. Section 2 develops a simplified version of the model of child quantity and time cost of children of Galor and Weil (1996). Section 3 characterises the conditions for the existence of stationary equilibria and studies equilibrium dynamics by clarifying the main theoretical results with simulative exercises (global analysis). Section 4 outlines the conclusions.

\footnotetext{
${ }^{1}$ See Krugman (1991) and Matsuyama (1991) for a debate about history versus self-fulfilling expectations. We recall here that models are said to be history driven when the initial conditions of an economy affect its long-term outcome. Alternatively, they are expectations driven when the agents' expectations about some macroeconomic aggregates are relevant in solving the decision-making problems and affect the long-term outcome of the economy.
} 


\section{The model}

This section builds on a modified version with homogeneous agents of the model of Galor and Weil (1996). The OLG (general equilibrium) closed economy is populated by a continuum of (perfectly) rational and identical individuals of size $N_{t}$ per generation $(t=0,1,2, \ldots)$. The life of the typical agent is divided into childhood and adulthood. As a child, an individual does not make economic decisions and spends time in the parent's household by consuming resources directly from him. As an adult, an individual is economically active, works and takes care of children when he is young, and retires when he is old. The $N_{t}$ members of generation $t$ overlap for one period (youth) with $N_{t-1}$ old individuals of generation $t-1$ and for one period (old-age) with $N_{t+1}$ young individuals of generation $t+1$. When young, an individual is endowed with 2 units of time. ${ }^{2}$ We assume that raising children is a purely time-consuming activity [see Guryan et al. (2008) for empirical evidence]. The child rearing technology requires an exogenous fraction $q>0$ of the parent's time endowment to raise a child that represents parent's foregone earnings, i.e. the time required to care for children cannot be spent working. Then, by letting $n_{t}>0$ be the number of children at time $t, q n_{t}$ is the time needed to care for $n_{t}$ descendants of a parent that belongs to generation $t$. This implies that the marginal time cost of children is constant, i.e. the opportunity cost of children is proportional to the wage rate. The remaining share $\ell_{t}=2-q n_{t}>0$ of time is supplied to firms in exchange for wage $w_{t}$ per unit of labour. Individuals consume only in the second period of life. The budget constraint of the young individual representative of generation $t$ is $s_{t}=w_{t} \ell_{t}$, i.e. labour income is entirely saved $\left(s_{t}\right)$ to consume one period later. When old, an individual retires and consumption $\left(C_{t+1}\right)$

\footnotetext{
${ }^{2}$ Considering a time endowment larger than one allows obtaining a growth factor of population larger than, smaller than or equal to one, i.e. a population that grows, decreases or it is stationary over time.
} 
is determined by the amount of resources saved when young plus expected interest accrued from time $t$ to time $t+1$ so that $C_{t+1}=R_{t+1}^{e} s_{t}$, where $R_{t+1}^{e}$ is the expected interest factor (realised at time $t+1)$. Therefore, the lifetime budget constraint can be expressed as follows:

$$
C_{t+1}=R_{t+1}^{e} w_{t}\left(2-q n_{t}\right)
$$

where $q n_{t}<2$ must hold to satisfy the constraint dictated by the individual time endowment.

An adult individual of generation $t$ has preferences towards the number of children and material consumption. Parents are selfish and give birth to children not for being supported when they will be old or enjoying their well-being but exclusively to increase their own utility. ${ }^{3}$ The lifetime utility of the individual representative of generation $t$ is given by the additively separable function (aimed for generality):

$$
U_{t}\left(n_{t}, C_{t+1}\right)=\frac{n_{t}^{1-\gamma}}{1-\gamma}+\frac{C_{t+1}^{1-\sigma}}{1-\sigma}
$$

where $\gamma>0(\gamma \neq 1)$ and $\sigma>0(\sigma \neq 1)$ are the constant elasticity of marginal utility with respect to fertility and consumption, respectively. In the particular case $\gamma=1$ and $\sigma=1$, the expression in (2) boils down to $U_{t}\left(n_{t}, C_{t+1}\right)=\ln \left(n_{t}\right)+\ln \left(C_{t+1}\right)$. The formulation for lifetime utility expressed in (2) is crucial for the results of this article and, in the case of no young material consumption, $1 / \sigma$ (resp. $1 / \gamma$ ) may be interpreted as a proxy for measuring the (constant) elasticity of substitution in consumption (resp. children). An increase in $\sigma$ (resp. $\gamma$ ) causes a decline in the marginal utility of material consumption (resp. fertility) when $C_{t+1}\left(\right.$ resp. $\left.n_{t}\right)$ increases. Empirical evidence [Hall (1988); Jones and Schoonbroodt (2010); Havranek et al. (2015)] finds that the elasticity of substitution in consumption is consistently smaller than one $(\sigma>1)$. Differently, $1 / \gamma$ can be interpreted as an index to measure the

\footnotetext{
${ }^{3}$ There exist several methodological approaches to model individual fertility choices and the debate is still open [see Ehrlich and Lui (1997) and Galor (2011)].
} 
elasticity of (inter-generational) substitution between consumption and children. In a recent work, Córdoba and Ripoll (2016) find that this index is significantly larger than one $(\gamma<1)$, meaning that material goods and children tend to be substitutes over time.

By substituting out (1) in (2) and taking factor prices as given, the maximisation problem of the individual representative of generation $t$ is:

$$
\max _{n_{t} \in(0,2 / q)}\left\{\frac{n_{t}^{1-\gamma}}{1-\gamma}+\frac{\left[R_{t+1}^{e} w_{t}\left(2-q n_{t}\right)\right]^{1-\sigma}}{1-\sigma}\right\} .
$$

Therefore, the first order condition is given by:

$$
n_{t}^{-\gamma}=q\left(R_{t+1}^{e} w_{t}\right)^{1-\sigma}\left(2-q n_{t}\right)^{-\sigma}
$$

Eq. (4) implies that the marginal utility of an extra child should be equal to the (indirect) marginal utility of material consumption. It tells us how much consumption to give up when old to consume one more child when young by keeping utility unaltered. The expression in (4) can also be rewritten as follows:

$$
\frac{n_{t}^{\gamma}}{\left(2-q n_{t}\right)^{\sigma}}=\frac{\left(R_{t+1}^{e} w_{t}\right)^{\sigma-1}}{q}
$$

An increase in the wage causes a twofold effect. On one hand, it implies that children become more costly relative to material consumption. Then, at the optimum, an individual wants to substitute the consumption of children when young for the consumption of material goods when old (substitution effect). On the other hand, an individual gets richer as the value of his overall time endowment increases (income effect). As he offers a positive amount of his time endowment to firms (labour supply), what eventually determines the sign of the change in the demand for children following a wage increase is the sign of the income effect. If a child is a normal (resp. inferior) good, then the income effect is positive (resp. negative). Therefore, an increase in wage 
income increases (resp. reduces) the demand for children through this channel. ${ }^{4}$ The first order condition in (5) allows clarifying the effects (at the individual level) of a change in wage income on the demand for children. In particular, the left-hand side of (5) is an increasing function of $n_{t}$. The right-hand side of (5) is an increasing (resp. decreasing) function of $w_{t}$ if $\sigma>1$ (resp. $\sigma<1$ ). When $\sigma>1$ (resp. $\sigma<1$ ) the income effect is positive (resp. negative) and children are a normal (resp. inferior) good. Therefore, if $\sigma>1$ an increase in wage income monotonically increases the demand for children and the income effect dominates the substitution effect. If $\sigma<1$ an increase in wage income monotonically reduces the demand for children and the income effect strengthens the negative substitution effect. Finally, if $\sigma=1$ fertility is $n=1 / q$, that is the demand for children is constant and independent of wage income [Galor (2012)]. This means that the substitution and income effects cancel exactly each other out and children are neither normal nor inferior goods. Therefore, depending on the value of $\sigma$ an additively separable utility function as the one expressed in (2) is capable of encompassing the different behaviours of individual fertility when wage income changes. This adds a novel utility-driven mechanism that can potentially explain the historical pattern of the fertility transition within the wider phenomenon of the demographic transition. The analysis of the relationship between fertility and GDP will be clarified later in this article in both cases $\sigma>1$ and $\sigma<1$ when we will account for the macroeconomic (general equilibrium) effects of the model.

Firms are identical and act competitively on the market. At time $t$, the representative firm produce a homogeneous good $\left(Y_{t}\right)$ by combining capital $\left(K_{t}\right)$ and labour $\left(L_{t}\right)$ by means of the

\footnotetext{
${ }^{4}$ The substitution effect and the income effect are of opposite sign and the final effect of a change in wage income on the demand for children is a priori uncertain when children are normal goods. The substitution effect and the income effect are both negative and the demand for children reduces when the wage increases (accord to the Beckerian tradition) when children are inferior goods.
} 
neoclassical technology:

$$
Y_{t}=A F\left(K_{t}, L_{t}\right)=A K_{t}^{\alpha} L_{t}^{1-\alpha}
$$

where $0<\alpha<1$ is the output elasticity of capital and $A>0$ is the (constant) productivity parameter. Profits are given by $A K_{t}^{\alpha} L_{t}^{1-\alpha}-w_{t} L_{t}-R_{t} K_{t}$. The temporary equilibrium condition in the labour market is $L_{t}=\ell_{t} N_{t}=\left(2-q n_{t}\right) N_{t}$, i.e. the amount of labour hired by firms is equal to the mass of young individuals of generation $t$ times the fraction of time they spend working. By taking factor prices as given, profit maximisation gives:

$$
\begin{gathered}
w_{t}=(1-\alpha) A k_{t}^{\alpha}\left(2-q n_{t}\right)^{-\alpha}, \\
R_{t}=\alpha A k_{t}^{\alpha-1}\left(2-q n_{t}\right)^{1-\alpha},
\end{gathered}
$$

where $k_{t}:=K_{t} / N_{t}$ is the stock of capital per young person.

The market-clearing condition in the capital market is $K_{t+1}=S_{t}:=s_{t} N_{t}$. As $N_{t+1}=n_{t} N_{t}$ determines the evolution of fertility, equilibrium reads as:

$$
k_{t+1}=\frac{s_{t}}{n_{t}},
$$

where $s_{t}=w_{t}\left(2-q n_{t}\right)$ and $n_{t}$ is determined by the individual first order conditions. By using (4), (7), (8), (9) and knowing that individuals have perfect foresight, so that $R_{t+1}^{e}=R_{t+1}=$ $\alpha A k_{t+1}^{\alpha-1}\left(2-q n_{t+1}\right)^{1-\alpha}$, the dynamics of the economy is characterised by the two-dimensional map:

$$
M:\left\{\begin{array}{l}
k_{t+1}=Q_{1}\left(k_{t}, n_{t}\right):=\frac{A(1-\alpha) k_{t}^{\alpha}\left(2-q n_{t}\right)^{1-\alpha}}{n_{t}} \\
n_{t+1}=Q_{2}\left(k_{t}, n_{t}\right):=\frac{1}{q}\left(2-k_{t}^{\frac{-\alpha^{2}}{1-\alpha}}\left(2-q n_{t}\right)^{-\alpha+\frac{1}{(1-\sigma)(1-\alpha)}} n_{t}^{-1-\frac{\gamma}{(1-\sigma)(1-\alpha)}} B\right)
\end{array},\right.
$$

defined on the set $X:=(0,+\infty) \times(0,2 / q)$, where

$$
B:=A^{\frac{-(1+\alpha)}{1-\alpha}}(1-\alpha)^{\frac{-\alpha}{1-\alpha}} \alpha^{\frac{-1}{1-\alpha}} q^{-\frac{1}{(1-\sigma)(1-\alpha)}} .
$$


For mathematical and economic reasons, in the reminder of the article we will concentrate on the study of the dynamics that starting from $X$ will remain in $X$ for every iterate. We note that capital per young person (resp. fertility) is a state (resp. control) variable and the initial value $n_{0}$ does not affect the initial value $k_{0}:=K_{0} / N_{0}$. The assumptions of additively separable preferences and perfect foresight are crucial and contribute to determine a dynamic expression for the number of children. In fact, if $\gamma=1$ and $\sigma=1$ (log-utility) fertility is constant and given by $n=1 / q$ so that the dynamics of the economy is characterised by the one-dimensional map $k_{t+1}=q(1-\alpha) A k_{t}^{\alpha}$, from which one can get the unique (globally asymptotically stable) stationary equilibrium $k^{*}=[q(1-\alpha) A]^{\frac{1}{1-\alpha}}$.

\section{Existence of stationary equilibria and equilibrium dy-}

\section{namics}

The analysis begins with by identifying the stationary equilibria of the map. By the first equation of map $M$ we have that at the stationary state it must hold:

$$
k=h(n):=(2-q n)\left(\frac{A(1-\alpha)}{n}\right)^{\frac{1}{1-\alpha}} .
$$

By using (12) and the second equation of system (10), the steady-state values of fertility are solutions of the following equation:

$$
n=g(n):=\frac{1}{q}\left(2-n^{\frac{2 \alpha-1}{(1-\alpha)^{2}}+\frac{\gamma}{(\sigma-1)(1-\alpha)}}(2-q n)^{-\frac{\alpha}{1-\alpha}-\frac{1}{(\sigma-1)(1-\alpha)}} D\right)
$$

where $D:=B[(1-\alpha) A]^{-\frac{\alpha^{2}}{(1-\alpha)^{2}}}$. From the expression in (12) it follows a negative relationship between $n$ and $k$ at the stationary state. This implies that larger values of the capital stock are related to lower values of fertility. Therefore, when there exist two stationary state equilibria 
$\left(k^{*}, n^{*}\right)$ and $\left(k^{* *}, n^{* *}\right)$, where $k^{*}<k^{* *}$, then $n^{*}>n^{* *}$ and the corresponding stationary state equilibrium values of production are $y^{*}<y^{* *}$. This is because when fertility is large, the time devoted for child bearing is higher than when fertility is small, so that parents reduce the labour supply. Then, under the assumption that children are a normal good $(\sigma>1)$ the model overcomes the paradox between individual choices and macro behaviour. In fact, the literature [see Macunovich (1998) and the works cited therein] has pinpointed the existence of a positive relationship between wage income and fertility at the individual level (the so-called Easterlin hypothesis), whereas larger values of GDP are associated with lower fertility in the last stages of the economic and demographic transition. ${ }^{5}$ Indeed, the former result holds despite the assumption of homogeneous agents, i.e. without distinguishing between male and female income, and it is due to the specific form of additively separable utility function (2). In fact, as is clear from (5) when $\sigma>1$ the income effect dominates the substitution effect.

In order to characterise the number of equilibria, we now study the behaviour of $g$ when $n \rightarrow 0^{+}$and when $n \rightarrow(2 / q)^{-}$.

\section{Lemma 1 Let}

$$
\widetilde{\gamma}:=\frac{(1-2 \alpha)(\sigma-1)}{1-\alpha}
$$

be threshold values of $\gamma$. (1) If $\sigma>1$ and $\gamma>\widetilde{\gamma}$ or if $\sigma<1$ and $\gamma<\widetilde{\gamma}$ then $\lim _{n \rightarrow 0^{+}} g(n)=2 / q$.

(2) If $\sigma>1$ and $\gamma<\widetilde{\gamma}$ or if $\sigma<1$ and $\gamma>\widetilde{\gamma}$ then $\lim _{n \rightarrow 0^{+}} g(n)=-\infty$. (3) If $\sigma>1$ then $\lim _{n \rightarrow(2 / q)^{-}} g(n)=-\infty$. (4) If $\sigma<1$ then $\lim _{n \rightarrow(2 / q)^{-}} g(n)=2 / q$.

\footnotetext{
${ }^{5}$ Although assessing the effects of changes in fertility on growth is not an easy task (this is because population variables change endogenously along the process of development), there exists evidence confirming the importance of fertility declines for explaining GDP growth [Palivos (1995); Jones and Tertilt (2006); Ashraf et al. (2013)].
} 
In addition, $g$ admits

$$
n_{c r i t}:=\frac{(4 \sigma-2 \gamma-4) \alpha-2 \sigma+2 \gamma+2}{\left[\alpha^{2}(\sigma-1)+(\sigma-\gamma) \alpha-\sigma+\gamma\right] q},
$$

as its unique critical point in the interval $(0,2 / q)$ if and only if $\gamma<\widetilde{\gamma}$. Otherwise, no critical points for $g$ exist in $(0,2 / q)$.

If

$$
[(1-\alpha) \gamma+2 \alpha \sigma-2 \alpha-\sigma+1][(1-\alpha) \gamma+3 \alpha \sigma-2 \alpha-2 \sigma+1](1-\sigma)>0
$$

then at most two inflection points for $g$ can exist in the interval $(0,2 / q)$.

Proof. Results (1)-(4) are obtained by studying the sign of the exponents of the terms $n$ and $2-q n$ in $g(n)$. Results on the critical point and the inflection points are obtained by noting that the zeros of $g^{\prime}(n)$ coincide with the zeros of the first degree polynomial:

$$
p(n):=q\left[\sigma\left(\alpha^{2}+\alpha-1\right)-(\alpha-1) \gamma-\alpha^{2}\right] n+2(\sigma-1)(1-2 \alpha)-2 \gamma(1-\alpha),
$$

and the zeros of $g^{\prime \prime}(n)$ coincide with the zeros of the second degree polynomial:

$$
P(n):=P_{2} n^{2}+P_{1} n+P_{0}
$$

where

$$
\begin{gathered}
P_{2}:=q^{2}\left[\left(\sigma-\frac{1}{3} \gamma-\frac{2}{3}\right) \alpha-\frac{2}{3} \sigma+\frac{1}{3} \gamma+\frac{1}{3}\right]\left[(\sigma-1) \alpha^{2}+(\sigma-\gamma) \alpha-\sigma+\gamma\right], \\
P_{1}:=-\frac{4}{3} q[(1-\alpha) \gamma+2 \alpha \sigma-2 \alpha-\sigma+1][(1-\alpha) \gamma+3 \alpha \sigma-2 \alpha-2 \sigma+1], \\
P_{0}:=-\frac{4}{3}[(1-\alpha) \gamma+2 \alpha \sigma-2 \alpha-\sigma+1]\left[(\sigma-1) \alpha^{2}+(-4 \sigma+\gamma+4) \alpha+2 \sigma-\gamma-2\right] .
\end{gathered}
$$

From the computation of the discriminant of (18), the result in (16) follows after some algebraic manipulations. 
Remark 2 We note that $\widetilde{\gamma}$ can be positive or negative. In particular, it is positive if and only if (a) $\sigma>1$ and $\alpha<1 / 2$, or (b) $\sigma<1$ and $\alpha>1 / 2$.

From Lemma 1, we get the next result characterising the existence and number of stationary equilibria of map $M$.

Proposition 3 [Existence and number of stationary equilibria]. (1) If $\sigma>1$ and $\gamma>\widetilde{\gamma}$ then there exists a unique interior fixed point. (2) If $\sigma>1$ and $\gamma<\widetilde{\gamma}$ [this case may arise if and only if $\alpha<1 / 2]$ then there exists a threshold value $\widetilde{A}>0$ such that for $A<\widetilde{A}$ there are no interior fixed points and for $A>\widetilde{A}$ there exist two interior fixed points. (3) If $\sigma<1$ and $\gamma>\widetilde{\gamma}$ then there exists a unique interior fixed point. (4) If $\sigma<1$ and $\gamma<\widetilde{\gamma}$ [this case may arise if and only if $\alpha>1 / 2]$ then there exists a threshold value $\bar{A}>0$ such that for $A<\bar{A}$ there exist two interior fixed points and for $A>\bar{A}$ there are no interior fixed points.

Proof. We separate the proof with respect to the cases introduced in the statement of the proposition.

(1) By Lemma 1, $g^{\prime}(n)$ has constant and negative sign in the interval $(0,2 / q)$. In fact, given

$$
\widehat{\gamma}:=\left[\alpha^{2}(\sigma-1)+\sigma(\alpha-1)\right] /(\alpha-1),
$$

we have that if $\gamma<\widehat{\gamma}$ then $p(n)$ defines a negatively sloped linear function that vanishes at a point $n<0$, whereas if $\gamma>\widehat{\gamma}$ then $p(n)$ defines a positively sloped linear function that vanishes at a point $n>2 / q$.

(2) By Lemma 1, $g$ has an interior maximum point $n_{\max }=n_{\text {crit }}$, and by the study of $g^{\prime \prime}(n)$ it follows that no inflection points exist and then $g$ is always concave.

(3) Function $g$ is increasing in the interval $(0,2 / q)$ and $\lim _{n \rightarrow 0^{+}} g(n)=-\infty, g(2 / q)=2 / q, g^{\prime}(2 / q)=$ 
0 (then, the graph of $g$ lies above the $45^{\circ}$ line in a left neighbourhood of $2 / q$ ). Due to the concavity of $g$, in a right (resp. left) neighbourhood of 0 (resp. $2 / q$ ) the number of inflection points is 0 or 2 . We will now verify that there are no inflection points in the interval $(0,2 / q)$. The expression in (16) is the product of three terms. Based on the assumptions on $\sigma$ and $\gamma$, it follows that the first two terms are negative and the third one is positive. The second term contributes to define (together with $-2 / q$ and $H$ ) the abscissa of the vertex $n_{V}$ of the parabola $P(n)=0$, where $P(n)$ is the expression in (18). Indeed, we have that:

$$
n_{V}:=-\frac{2}{q} \frac{(\gamma-2 \sigma+2) \alpha+\sigma-\gamma-1}{H}
$$

where

$$
H:=(\sigma-1) \alpha^{2}-(\sigma-\gamma)(1-\alpha) \text {. }
$$

If $H<0$ then $n_{V}<0$, so that at least one root of $P(n)=0$ is negative, but this implies that also the other root does not belong to $(0,2 / q)$. If $H>0$, it is straightforward to verify that $n_{V}>2 / q$, so that at least one root of $P(n)=0$ is larger than $2 / q$, but this implies that the two roots of $P(n)=0$ do not belong to $(0,2 / q)$.

(4) Function $g$ always admits a minimum point $n_{\min }$ in the interval $(0,2 / q)$. We note that $\lim _{n \rightarrow 0^{+}} g^{\prime}(n)=\lim _{n \rightarrow 0^{+}}-n^{\frac{(\sigma-1)\left(\alpha^{2}-4 \alpha+2\right)-\gamma(1-\alpha)}{\left(1-\alpha^{2}\right)(1-\sigma)}}$. Then, depending on the sign of $(\sigma-1)\left(\alpha^{2}-4 \alpha+\right.$ $2)-\gamma(1-\alpha)$ it is possible to classify two cases. Indeed, let us define the threshold $\bar{\gamma}:=$ $\left(\alpha^{2}-4 \alpha+2\right)(\sigma-1) /(1-\alpha)$, where $\bar{\gamma}<\widetilde{\gamma}$. If $\bar{\gamma}<\gamma<\widetilde{\gamma}$ then $g(0)=g(2 / q)=2 / q, g^{\prime}(2 / q)=0$ and $\lim _{n \rightarrow 0^{+}} g^{\prime}(n)=-\infty$. From a geometrical reasoning, it follows that $g$ has a unique inflection point $f_{1}$ in the interval $(0,2 / q)$, with $n_{\text {crit }}<f_{1}$ and $g$ is decreasing and convex in the interval $\left(0, n_{\min }\right)$, where $n_{\min }:=n_{\text {crit }}$, and $g$ is increasing and convex in the interval $\left(n_{\min }, f_{1}\right)$, it is increasing and concave in the interval $\left(f_{1}, 2 / q\right)$, it eventually ends up at point $2 / q$. If $\gamma<\bar{\gamma}$ then $g(0)=g(2 / q)=2 / q$ and $g^{\prime}(2 / q)=\lim _{n \rightarrow 0^{+}} g^{\prime}(n)=0$. From a geometrical reasoning, it 
follows that $g$ has two inflection points $f_{1}$ and $f_{2}$ in the interval $(0,2 / q)$, with $f_{1}<n_{\text {crit }}<f_{2}$, and $g$ is decreasing and concave in the interval $\left(0, f_{1}\right)$, it is decreasing and convex in the interval $\left(f_{1}, n_{\min }\right)$, it is increasing and convex in the interval $\left(n_{\min }, f_{2}\right)$, it is increasing and concave in the interval $\left(f_{2}, 2 / q\right)$ with $g(2 / q)=2 / q$ and $g^{\prime}(2 / q)=0$.

Results on threshold values of $A$ in defining the number of equilibria at Points (2) and (4) can be obtained by noting that the value of $g(n)$ is an increasing function of $A$ for any $n \in(0,2 / q)$.

The geometry of existence and number of stationary states of map $M$ outlined in Proposition 3 is illustrated in Panels (a)-(d) of Figure 1. The stationary states are the intersection points of $g(n)$ with the $45^{\circ}$ line. Depending on parameter configurations, there exists either uniqueness (Panels (a) and (c)) or multiplicity (Panels (b) and (d)) of steady states. By concentrating on the role of $A$, it is possible to show (by applying the implicit function theorem to the solutions of Eq. (13)) that an increase in $A$ causes: (a) an increase (resp. a decrease) in the stationarystate value of $n$ under the hypothesis of Point (1) (resp. Point (3)) of Proposition 3; (b) an increase (resp. decrease) in the gap between the stationary states under the hypothesis of Point (2) (resp. Point (4)) of Proposition 3. Though the role of $A$ in defining the stationary-state value of $n$ is clear (according to the configurations defined in Proposition 3), its effects on the stationary-state value of $k$ are much more articulated. In fact, when an increase in $A$ causes a reduction in the stationary-state value of $n$, it also causes an increase in the corresponding value of $k$. On the other hand, when an increase in $A$ implies an increase in the stationary-state value of $n$, the corresponding stationary-state value of $k$ may increase or decrease depending on the parameter values.

[FIGURE 1 ABOUT HERE] 
Remark 4 Compared to the cases studied in Proposition 3, we note that from an empirical point of view $\alpha<0.5$ [Krueger (1999); Gollin (2002); Jones (2004)] and $\sigma>1$ [Hall (1988); Jones and Schoonbroodt (2010); Havranek et al. (2015)] generally hold. Values of the capital share in income larger than 0.5 may make sense only by broadening the concept of capital including human components [see Chakraborty (2004) and the literature cited therein]. The numerical simulations of this work adopt the usual notion of physical capital and make use of a standard value of $\alpha$ around 0.33 , which is consistent with the data, and are also in line with the assumption that $n$ is a normal good $(\sigma>1)$.

We now focus on a situation where there exist two stationary states under the assumption that children are a normal good $(\sigma>1)$. In the long term, workers will get a lower wage at a stationary state with a low stock of capital (less developed economy) in comparison with the wage that will be obtained at a stationary state where capital is larger (developed economy). Given the same interest factor (partial equilibrium), this means that individuals choose to have less children in poor countries than in richer countries. However, the model also implies that the interest factor is higher where the capital stock is lower. As children are a normal good and the interest factor is an element that helps capitalising wage income over time, in the long term (general equilibrium) fertility increases when the capital stock reduces. Then, this theory is able to explain the behaviour of the demand for children both at microeconomic and macroeconomic levels.

Of course, the existence of one or more stationary states is economically meaningful only whether there exist trajectories leading to convergence. Now, let $\gamma_{1}:=\frac{\sigma\left(2-q n_{s s}\right)-2}{2-q n_{s s}}$ and $\gamma_{2}:=$ $\frac{(\sigma-1)\left(\alpha^{2} q n_{s s}+2 \alpha+2\right)+q n_{s s} \sigma}{2-q n_{s s}}$, where $\gamma_{2}>\gamma_{1}$. The following results provide a classification of the equilibrium dynamic properties of map $M$. 
Lemma 5 If (a) $\sigma<1$ and $\gamma>\sigma$ or (b) if $\sigma>1$ and $\gamma>\gamma_{1}$ then the determinant of the Jacobian matrix of map $M$ is positive, where $n_{\text {ss }}$ is the generic stationary state value of $n$.

Proposition 6 [Local stability of stationary equilibria]. Under the hypotheses of Lemma 5, if the graph of $g$ at $n_{s s}$ intersects the $45^{\circ}$ line from below, the point $\left(k_{s s}, n_{s s}\right)$ is a saddle, where $k_{s s}$ is the generic stationary state value of $k$ obtained by the expression in (12).

Proposition 7 If (a) $\sigma<1$ or (b) $\sigma>1$ and $\gamma_{1}<\gamma<\gamma_{2}$. If the graph of $g$ at $n_{s s}$ intersects the $45^{\circ}$ line from above, the point $\left(k_{s s}, n_{s s}\right)$ is not a saddle.

Proof. The proof of Lemma 5 and Propositions 6 and 7 are in the Appendix.

In the light of the previous results, we can give an insight about the stability of equilibrium points in the different cases outlined in Figure 1. Specifically, under the assumptions introduced in the propositions, the left-located stationary state in Panel (b) identifies a saddle point. This means that given an initial condition on the stock of capital close to its value at the saddle point, there exists a unique choice on the control variable allowing the economy to lie on the trajectory converging towards it. The same result holds for the unique stationary state identified in Panel (c) and for the right-located one of Panel (d). Instead, nothing can be said in the case detailed in Panel (a) and in regard to the left-located stationary state of Panel (d), as equilibrium dynamics can have different properties.

Local indeterminacy. In order to clarify the outcome in the former case, Figure 2 shows the possibility that the equilibrium is locally indeterminate. Although this is the unique stationary state of the model, there exist infinite choices on the control variable (fertility) generating trajectories that lead towards it for a given initial condition of the state variable (capital). These trajectories are characterised by different values of capital accumulation and fertility. 
In other words, every choice of $n$ that identifies a point within the grey region of Figure 2 , generates a trajectory convergent towards the fixed point (the black dot in the figure).

\section{[FIGURE 2 ABOUT HERE]}

When the hypotheses of the previous results are violated, the classification of the stationary states of map $M$ is quite cumbersome from an analytical point of view, with conditions that are very difficult to be interpreted economically. More details are available in the Appendix.

Global indeterminacy. As is shown in Proposition 3, it is possible to have multiple stationary states. This implies that the model is able to generate distinct development trajectories leading to different long-term values of capital (state variable) and fertility (choice variable). This makes it also possible to have global indeterminacy. For instance, by using a meaningful parameter set (Figure 3), we obtain two stationary states. One of them is a locally indeterminate fixed point and represents the long-term under-development regime (low GDP and high fertility). This means that every choice about fertility that identifies a point in the grey region of Figure 3 , defines a trajectory convergent towards $\left(k^{*}, n^{*}\right)=(1.23,1.13)$. The other one is a saddle and represents the long-term developed regime (high GDP and low fertility). It is given by $\left(k^{* *}, n^{* *}\right)=(1.96,0.88)$. Therefore, values of $k$ ranging from about 1.01 to about 1.78 can generate trajectories that converge towards distinct fixed points. It is important to note that only a sufficiently large value of $k$ can guarantee that the economy lies in a development trajectory with high GDP and low fertility (history matters).

\section{[FIGURE 3 ABOUT HERE]}


On the existence of feasible trajectories with $k_{t} \rightarrow 0$ and $n_{t} \rightarrow 2 / q$ when $\sigma<1$. By exploring the two equations of map $M$, it is not possible to have feasible trajectories such that $k_{t} \rightarrow 0$ and $n_{t} \rightarrow 2 / q$ when $\sigma<1$. In this case, point $(0,2 / q)$ is an attractor of the system even though the map is not defined on such a point. This event is shown through numerical simulations in the example of Figure 4. Panel (a) depicts the (grey-coloured) basin of attraction of $(0,2 / q)$, depicted in red in the figure, whereas the boundary of the basin is defined by the stable manifold of the interior stationary state, i.e. the saddle (black) point $\left(k^{*}, n^{*}\right)$. The white region represents the space of initial conditions for which trajectories become unfeasible after a finite number of iterations. From Figure 4(a), one may conclude that the system is globally indeterminate as there exist two distinct long-term outcomes (only one of them is an interior fixed point) that can be achieved given the same initial condition. Specifically, there exist (1) an infinite number of trajectories leading to the locally indeterminate state $(0,2 / q)$, which represents a poverty trap scenario with a low level of capital and high fertility, and (2) a unique (saddle) path on which the economy converges to the interior stationary state, which represents a paradigm of developed countries with a high level of capital and low fertility. Converging towards one of these long-term state is a matter of individuals' choices about fertility. This is a typical expectations-driven outcome leading to coordination failures. In fact, $U$ evaluated at $(0,2 / q)$ is smaller than $U$ evaluated at $\left(k^{*}, n^{*}\right)$ meaning that $\left(k^{*}, n^{*}\right)$ Pareto dominates $(0,2 / q)$ but individuals can choose to coordinate themselves on the Pareto dominated equilibrium. This holds because individuals (by expecting a low return on capital) tend to increase the amount of time devoted to child rearing. Then, fertility increases approaching its upper bound $(2 / q)$ and capital increasingly reduces. Panel (b) of Figure 4 shows two typical trajectories leading to the poverty trap outcome. 


\section{[FIGURE 4 ABOUT HERE]}

Endogenous fluctuations. Another result the model is able to reproduce is given by the endogenous fertility fluctuations ${ }^{6}$ that are in line with the baby busts and baby booms observed the last century in some developed countries. There are two recent articles analysing the reasons why fertility fluctuates over time. We refer to the works of Doepke et al. (2015) and Jones and Schoonbroodt (2016). The former studies the effects of the shock of World War II on subsequent baby boom in U.S. (following the historical decrease in fertility due to working of demographic transition forces). The author considers a model where women can choose the labour supply, the number of children, when having children and there exists an interaction amongst subsequent cohorts. Then, they perform quantity experiments to explain the postwar increase in fertility based on a drop in labour force participation of young women (whose wages declined in that period) because of the increase in competition caused by the higher participation of older women (and the persisted high demand of female labour after the end of World War II). Then, younger women exited the labour market and started having children. The latter work, instead, considers a general equilibrium model with endogenous fertility and dynastic altruism showing that fertility and the opportunity cost of children in U.S. are procyclical. Our theory simplifies the framework substantially and is able to explain fertility

\footnotetext{
${ }^{6}$ From a mathematical point of view, a stationary state equilibrium in this model can undergo either a flip bifurcation (see, for instance, the time series in Figure 5 where fluctuations are governed by an attractor generated by a sequence of flip bifurcations) or a Neimark-Sacker bifurcation. For this second type of bifurcation see Figure 3, where the basin of attraction of $\left(k^{*}, n^{*}\right)$ is bounded by an invariant curve generated by a subcritical Neimark-Sacker bifurcation. We note that in the present model where there exists a control variable $\left(n_{t}\right)$, the sub-critical bifurcation is important not only to define the boundary of a basin of attraction but also to characterise the possible dynamics of the model. This is because agents can coordinate on this set.
} 
fluctuations within a typical neoclassical set up trading off between fertility and labour supply (see Figure 5 for a simulative exercise).

\section{[FIGURE 5 ABOUT HERE]}

\section{Conclusions}

For a very long time in human history the number of births and deaths were almost equivalent, and the world total population was relatively stable (fluctuating around a low level). During this extended period, income per person remained quite constant or grew slowly [Galor and Weil (2000)]. After the process commonly known as Industrial Revolution, mortality started declining in newly industrialised countries and after an initial stalling stage, fertility followed a declining trend as well. In these phases, total population and income marked the start of an increasing. Then, Europe faced a long age of dramatic social, political and institutional changes that subsequently spread to other regions all over the world.

Economic development is a long-term involved phenomenon that includes social, institutional, economic and demographic changes across nations and continents. The present work treated development on the side of economic and demographic transition. Why do some countries achieve high levels of GDP and low fertility and others low levels of GDP and high fertility? Standard one-sector models of neoclassical growth often conclude that economies with similar technologies will converge towards a common stationary-state equilibrium even if the initial conditions are very different. This is the main result of the Solow-type growth set up implying that poorer countries will growth faster than richer countries. This finding is also shared by several works in the OLG and continuous-time optimal growth literature. However, it was 
widely accepted that there exist persistent differences in the level of real activity and fertility rates amongst (distinct groups of) countries [Mankiw et al. (1992); Wang et al. (1994); Palivos (1995); Hall and Jones (1999); Jones and Tertilt (2006)]. This kind of models, therefore, cannot explain these macroeconomic and demographic differences, so that the above as well as other similar questions are likely to remain unanswered within both the basic neoclassical growth set up and endogenous growth one. This unsatisfactory result has led several economists to modify these frameworks in several ways trying to building on more suitable theories with exogenous fertility [Azariadis and Drazen (1990)] or endogenous fertility [Galor and Weil (1996)]. The literature has then grown rapidly leading to what is commonly known as the UGT [Galor (2011)], where the main factors explaining the demographic and economic transitions were generally child and adult mortality, human capital accumulation and structural changes. However, it is still difficult to find theories where fertility per se represents the triggering factor for the transition amongst the various stages of development. The present article used a basic OLG model of neoclassical growth to fill that gap. The works most closely related to ours are Palivos (1995) and Galor and Weil (1996). The former introduced endogenous fertility (child quantity and child quality) in a continuous-time neoclassical optimal growth set up with infinite lived individuals, finding a production-driven channel through which fertility choice may be a source of multiple steady states and coordination failures. The latter emphasised the importance of gender differences in wage income for the existence of multiple paths of economic development in an OLG model with child quantity. The present work showed that multiple paths of economic development can exist also in a modified version of Galor and Weil (1996) with homogeneous agents. It also introduced a new utility-driven mechanism through which fertility is a source of global indeterminacy. 
The work had the ambition of giving an answer to the question raised by Jones et al. (2008): "Fertility Theories: Can They Explain the Negative Fertility-Income Relationship?" Of course, we are aware that this is toy model and preferences may depend on culture, beliefs and social norms specifically related to institutions or ethnic groups (often followed by linguistic and religious contours that also affect choices about contraception), and that these elements should therefore be included as endogenous variables in the analysis. However, the main goal of the work was to keep the model as simple as possible to bring to light some theoretical outcomes that remained until now unexplored within the standard economic theory of fertility.

Acknowledgements The authors gratefully acknowledge Davide Fiaschi and conference participants at MDEF 2016 held at University of Urbino (Italy), CRISIS 2016 held at University of Ancona (Italy), AMASES 2016 held at University of Catania (Italy), NED-CICSE 2017 held at University of Pisa (Italy) and SIE 2017 held at University of Calabria (Italy) for valuable comments on an earlier draft. The authors are also grateful to two anonymous reviewers for valuable comments allowing an improvement in the general quality of the work. The usual disclaimer applies.

Conflict of Interest The authors declare that they have no conflict of interest.

\section{Appendix}

For the sake of completeness, we analyse here some mathematical properties of map $M$ not discussed in the main text.

On the feasible region of map $M$. Map $M$ is defined on a sub set of the non-negative orthant. 
In fact, given $n_{t}>0$ and $k_{t}>0$, in order to have $n_{t+1}>0$ and $k_{t+1}>0$ it must hold that

$$
k_{t}>\left[\frac{\left(2-q n_{t}\right)^{-\alpha-\frac{1}{(\sigma-1)(1-\alpha)}} n_{t}^{-1+\frac{\gamma}{(\sigma-1)(1-\alpha)}}}{2 B}\right]^{\frac{1-\alpha}{\alpha^{2}}}
$$

where $B:=A^{\frac{-(1+\alpha)}{1-\alpha}}(1-\alpha)^{\frac{-\alpha}{1-\alpha}} \alpha^{\frac{-1}{1-\alpha}} q^{-\frac{1}{(1-\sigma)(1-\alpha)}}$. Depending on the parameter setting we have three different cases.

1) If $\sigma>1$ and $\gamma>(\sigma-1)(1-\alpha)$ then the region defined by the inequality in (25) is described by the grey area in Panel (a) of Figure A.1.

2) If $\sigma>1$ and $\gamma<(\sigma-1)(1-\alpha)$ then the region defined by the inequality in (25) is described by the grey area in Panel (b) Figure A.1.

3) If $\sigma<1$ then the region defined by the inequality in (25) is described by the grey area in Panel (c) of Figure A.1.

The regions detailed above ensure the possibility of computing a single iterate. However, in order to have well-defined forward dynamics, the trajectory generated by a generic initial condition must be bounded in this region for every iterate. For this reason, the economically meaningful trajectories analysed in the main text actually lie on in a smaller region than the one shown in the three panels of Figure A.1.

\section{[FIGURE A.1 ABOUT HERE]}

The following results define some properties of the dynamic system. In particular, we will study the existence (or non-existence) of trajectories tending towards the boundaries of the feasible region. For the sake of clarity, we now report the stationary equilibrium conditions of 
the system:

$$
\left\{\begin{array}{l}
k=\frac{A(1-\alpha) k^{\alpha}(2-q n)^{1-\alpha}}{n} \\
n=\frac{1}{q}\left(2-k^{\frac{-\alpha^{2}}{1-\alpha}}(2-q n)^{-\alpha+\frac{1}{(1-\sigma)(1-\alpha)}} n^{-1-\frac{\gamma}{(1-\sigma)(1-\alpha)} B}\right)
\end{array}\right.
$$

On the non-existence of feasible trajectories with $n_{t} \rightarrow 2 / q$ when $\sigma>1$. We can rule out the existence of feasible trajectories with $n_{t} \rightarrow 2 / q$ in the case $\sigma>1$. If there were feasible trajectories such that $n_{t} \rightarrow 2 / q$ then by the first equation in (26) $k_{s s}=0$. However, this would not be consistent with the second equation in (26). We note that the result on the non-existence of feasible trajectories with $n_{t} \rightarrow 2 / q$ cannot be generalised to $\sigma<1$. In fact, in that case it is possible to find numerical specifications for which there exist feasible trajectories such that $n_{t} \rightarrow 2 / q$ and $k_{t} \rightarrow 0$. This case resembles the typical poverty trap scenario.

On the non-existence of feasible trajectories with $n_{t} \rightarrow 0$. Let us assume by contradiction that for $t \rightarrow+\infty$ there exists a trajectory with $n_{t} \rightarrow 0$. By the first equation in (26), we have that $k \simeq 2\left(\frac{A(1-\alpha)}{n}\right)^{\frac{1}{1-\alpha}}$. By substituting the last expression into the second equation in (26), we obtain a non-consistent expression.

On the non-existence of feasible trajectories with $k_{t} \rightarrow+\infty$. Let us assume by contradiction that for $t \rightarrow+\infty$ there exists a trajectory with $k_{t} \rightarrow+\infty$. By the first equation of map $M$, we have that $n_{t} \rightarrow 0$. Therefore, for $t \rightarrow+\infty$ by the first equation in (26) we have that $n \simeq \frac{2^{1-\alpha} A(1-\alpha)}{k^{1-\alpha}}$. By substituting the last expression in the second equation of (26), we obtain a non-consistent expression.

\section{On the stability properties of the fixed points}

In this section, we will examine some details about the stability properties of the fixed points of map (10) in the main text. Results about stability of the stationary states follow by the 
study of the Jacobian matrix evaluated at a generic state $\left(k_{s s}, n_{s s}\right)$. The Jacobian matrix is the following:

$$
J\left(k_{s s}, n_{s s}\right):=\left(\begin{array}{cc}
J_{1,1} & J_{1,2} \\
J_{2,1} & J_{2,2}
\end{array}\right)
$$

where

$$
\begin{gathered}
J_{11}:=\alpha>0 \\
J_{1,2}:=-[(1-\alpha) A]^{\frac{1}{1-\alpha}} n^{\frac{\alpha-2}{1-\alpha}}\left(2-\alpha q n_{s s}\right)<0, \\
J_{2,1}:=\frac{\alpha^{2} k_{s s}^{\frac{\alpha^{2}}{\alpha-1}}\left(2-q n_{s s}\right)^{\frac{\left(\alpha^{2}-\alpha\right)(1-\sigma)+1}{(1-\sigma)(1-\alpha)}} n_{s s}^{\frac{(1-\alpha)(\sigma-1)-\gamma}{(1-\sigma)(1-\alpha)}} B}{q(1-\alpha) k_{s s}}>0, \\
J_{2,2}:=\frac{J_{2,1} k_{s s}\left\{\left[\alpha^{2}(\sigma-1)-\sigma\right] q n_{s s}+2(\sigma-1)(1+\alpha)-\left(2-q n_{s s}\right) \gamma\right\}}{\alpha^{2}(\sigma-1)\left(2-q n_{s s}\right) n_{s s}},
\end{gathered}
$$

and

$$
\begin{aligned}
\operatorname{Det}\left(J\left(k_{s s}, n_{s s}\right)\right)= & \frac{2+(\gamma-\sigma)\left(2-q n_{s s}\right)}{1-\sigma} \frac{\alpha A B}{q} \times \\
& \times k_{s s}^{\frac{\alpha^{2}}{\alpha-1}+\alpha-1} n_{s s}^{-\frac{\alpha \sigma-\alpha+\gamma-\sigma+1}{(-1+\sigma)(\alpha-1)}-2}\left(2-n_{s s} q\right)^{-\frac{\alpha^{2} \sigma-\alpha^{2}-\alpha \sigma+\alpha-1}{(-1+\sigma)(\alpha-1)}-\alpha} .
\end{aligned}
$$

Proof of Lemma 5. Result follows from the study of the sign of $\operatorname{Det}\left(J\left(k_{s s}, n_{s s}\right)\right)$, where

$$
\operatorname{sgn}\left\{\operatorname{Det}\left(J\left(k_{s s}, n_{s s}\right)\right)\right\}=\operatorname{sgn}\left\{\frac{2+(\gamma-\sigma)\left(2-q n_{s s}\right)}{1-\sigma}\right\}
$$

As map (10) in the main text defines a two-dimensional dynamic system with one state variable and one control (or jump) variable, the stability properties can be studied in $\left(\operatorname{Tr}\left(J\left(k_{s s}, n_{s s}\right), \operatorname{Det}\left(J\left(k_{s s}\right.\right.\right.\right.$ plane. In particular, the results about determinacy/indeterminacy described in Grandmont et al. (1998) hold: a generic stationary state is a saddle (determinate) if

$$
\left|\operatorname{Tr}\left(J\left(k_{s s}, n_{s s}\right)\right)\right|>\left|1+\operatorname{Det}\left(J\left(k_{s s}, n_{s s}\right)\right)\right|,
$$


it is a source (completely unstable) if

$$
\left\{\begin{array}{c}
\left|\operatorname{Tr}\left(J\left(k_{s s}, n_{s s}\right)\right)\right|<\left|1+\operatorname{Det}\left(J\left(k_{s s}, n_{s s}\right)\right)\right| \\
\left|\operatorname{Det}\left(J\left(k_{s s}, n_{s s}\right)\right)\right|>1
\end{array}\right.
$$

it is a sink (indeterminate) if

$$
\left\{\begin{array}{c}
\left|\operatorname{Tr}\left(J\left(k_{s s}, n_{s s}\right)\right)\right|<1+\operatorname{Det}\left(J\left(k_{s s}, n_{s s}\right)\right) \\
\left|\operatorname{Det}\left(J\left(k_{s s}, n_{s s}\right)\right)\right|<1
\end{array} .\right.
$$

Proof of Proposition 6. As $g(n)=v(h(n), n)$ then $g^{\prime}(n)=v_{k}^{\prime}(h(n), n) h^{\prime}(n)+v_{n}^{\prime}(h(n), n)$. At a stationary state, this expression becomes:

$$
v_{k}^{\prime}\left(k_{s s}, n_{s s}\right) \frac{\left.\frac{\partial Q_{1}}{\partial n}\right|_{(k, n)=\left(k_{s s}, n_{s s}\right)}}{1-\left.\frac{\partial Q_{1}}{\partial k}\right|_{(k, n)=\left(k_{s s}, n_{s s}\right)}}+v_{n}^{\prime}\left(k_{s s}, n_{s s}\right) .
$$

Corresponding to an intersection from below (resp. above) of the graph of $g$ with the $45^{\circ}$ line, we have that $g^{\prime}(n)>1($ resp. $<1)$. By rearranging terms, the expression $g^{\prime}(n)>1($ resp. $<1)$ is equivalent to $\operatorname{Det}\left(J\left(k_{s s}, n_{s s}\right)\right)-\operatorname{Tr}\left(J\left(k_{s s}, n_{s s}\right)\right)+1<0$ (resp. $\left.>0\right)$. The result follows from the assumptions on the sign of the determinant.

Proof of Proposition \%. Due to the assumptions introduced in the statement of the proposition, the trace and determinant associated with a stationary point are both positive $\left(J_{2,2}>0\right)$. Therefore, the condition $g_{\prime}(n) \lessgtr 1$ discriminates between determinacy on one hand and indeterminacy/instability on the other.

\section{Comparative statics}

On the dependence of $n_{s s}$ on $A$. We note that the fixed points $\left(k_{s s}, n_{s s}\right)$ of map $M$ are such that $n_{s s}-g\left(n_{s s}\right)=0$, where

$$
g(n)=\frac{1}{q}\left(2-n^{\frac{2 \alpha-1}{(1-\alpha)^{2}}+\frac{\gamma}{(\sigma-1)(1-\alpha)}}(2-q n)^{-\frac{\alpha}{1-\alpha}-\frac{1}{(\sigma-1)(1-\alpha)}} A^{\frac{-1}{(1-\alpha)^{2}}}(1-\alpha)^{\frac{-\alpha}{(1-\alpha)^{2}}} \alpha^{\frac{-1}{1-\alpha}} q^{-\frac{1}{(1-\sigma)(1-\alpha)}}\right) .
$$


Let us now consider $G(n, A)=n-g(n)$. Then, it is possible to study the dependence of $n_{s s}$ on $A$ by using the implicit function theorem. We have that:

$$
\begin{aligned}
\frac{d n_{s s}}{d A} & =-\frac{\partial G\left(n_{s s}, A\right) / \partial A}{\partial G\left(n_{s s}, A\right) / \partial n}= \\
& =\frac{-n^{\frac{2 \alpha-1}{(1-\alpha)^{2}}+\frac{\gamma}{(\sigma-1)(1-\alpha)}}(2-q n)^{-\frac{\alpha}{1-\alpha}-\frac{1}{(\sigma-1)(1-\alpha)}}}{\left[g^{\prime}\left(n_{s s}\right)-1\right] q^{\frac{\alpha}{(1-\sigma)(1-\alpha)}+1}(1-\alpha)^{\frac{\alpha}{(1-\alpha)^{2}}+2} \alpha^{\frac{1}{1-\alpha}} A^{\frac{1}{(1-\alpha)^{2}}+1}} .
\end{aligned}
$$

Then, the sign of the numerator of the previous expression is always negative whereas the denominator is positive (resp. negative) corresponding to the fixed points for which the graph of $g$ intersects the $45^{\circ}$ line from below (resp. above). From the sign of $d n_{s s} / d A$ the results in the article follow.

On the dependence of $k_{s s}$ on $A$. We can use the implicit function theorem by generalising the approach used in the previous point. In particular, a fixed point $\left(k_{s s}, n_{s s}\right)$ of map $M$ is a solution of the following system:

$$
\left\{\begin{array}{c}
G_{1}(k, n, A):=k-(2-q n)\left(\frac{A(1-\alpha)}{n}\right)^{\frac{1}{1-\alpha}}=0 \\
G_{2}(k, n, A):=n-g(n)=0
\end{array} .\right.
$$

Then, we have that

$$
\left(\begin{array}{c}
\frac{d k_{s s}}{d A} \\
\frac{d n_{s s}}{d A}
\end{array}\right)=-J_{G_{1}, G_{2}}\left(\begin{array}{c}
\partial G_{1}\left(k_{s s}, n_{s s}, A\right) / \partial A \\
\partial G_{2}\left(k_{s s}, n_{s s}, A\right) / \partial A
\end{array}\right)
$$

where

$$
\begin{aligned}
J_{G_{1}, G_{2}} & :=\left(\begin{array}{cc}
\partial G_{1}\left(k_{s s}, n_{s s}, A\right) / \partial k & \partial G_{1}\left(k_{s s}, n_{s s}, A\right) / \partial n \\
\partial G_{2}\left(k_{s s}, n_{s s}, A\right) / \partial k & \partial G_{2}\left(k_{s s}, n_{s s}, A\right) / \partial n
\end{array}\right)^{-1}= \\
& =\frac{1}{1-g^{\prime}\left(n_{s s}\right)}\left(\begin{array}{cc}
1-g^{\prime}\left(n_{s s}\right) & -\partial G_{1}\left(k_{s s}, n_{s s}, A\right) / \partial n \\
0 & 1
\end{array}\right) .
\end{aligned}
$$


We note that $\partial G_{1}\left(k_{s s}, n_{s s}, A\right) / \partial n>0$. By direct computations, it follows that the dependence of $k$ on $A$ is not ambiguous in general if and only if $1-g^{\prime}\left(n_{s s}\right)<0$. This last inequality can be interpreted in the light of the graphs of Figure 1 in the main text of the article.

\section{References}

[1] Ashraf, Q.H., D.N. Weil and J. Wilde (2013) The effect of fertility reduction on economic growth. Population and Development Review 39, 97-130.

[2] Azariadis, C. and A. Drazen (1990) Threshold externalities in economic development. Quarterly Journal of Economics 105, 501-526.

[3] Becker, G.S. (1960) An economic analysis of fertility. In: Demographic and economic change in developing countries, National Bureau Committee for Economic Research, Princeton University Press, Princeton (NJ).

[4] Blackburn, K. and G.P. Cipriani (2002) A model of longevity, fertility and growth. Journal of Economic Dynamics \& Control 26, 187-204.

[5] Cervellati, M. and U. Sunde (2005) Human capital, life expectancy, and the process of development. American Economic Review 95, 1653-1672.

[6] Cervellati, M. and U. Sunde (2013) Life expectancy, schooling, and lifetime labor supply: theory and evidence revisited. Econometrica 81, 2055-2086.

[7] Cervellati, M. and U. Sunde (2015) The economic and demographic transition, mortality, and comparative development. American Economic Journal: Macroeconomics 7, 189-225. 
[8] Chakraborty, S. (2004) Endogenous lifetime and economic growth. Journal of Economic Theory 116, 119-137.

[9] Córdoba, J.C. and M. Ripoll (2016) Intergenerational transfers and the fertility-income relationship. Economic Journal 126, 949-977.

[10] Doepke, M. (2005) Child mortality and fertility decline: does the Barro-Becker model fit the facts? Journal of Population Economics 18, 337-366.

[11] Doepke, M., M. Hazan and Y.D. Maoz (2015) The baby boom and World War II: a macroeconomic analysis. Review of Economic Studies 82, 1031-1073.

[12] Ehrlich, I. and F. Lui (1997) The problem of population and growth: a review of the literature from Malthus to contemporary models of endogenous population and endogenous growth. Journal of Economic Dynamics and Control 21, 205-242.

[13] Fanti, L. and L. Gori (2014) Endogenous fertility, endogenous lifetime and economic growth: the role of child policies. Journal of Population Economics 27, 529-564.

[14] Fiaschi, D. and T. Fioroni (2014) Transition to modern growth: the role of technological progress and adult mortality. Discussion Papers no. 186, Department of Economics and Management, University of Pisa.

[15] Fogel, R.W. (2004) The escape from hunger and premature death. Cambridge University Press, New York (NY).

[16] Galor, O. (2011) Unified growth theory. Princeton University Press, Princeton (NJ).

[17] Galor, O. (2012) The demographic transition: causes and consequences. Cliometrica 6, $1-28$. 
[18] Galor, O. and O. Moav (2002) Natural selection and the origin of economic growth. Quarterly Journal of Economics 117, 1133-1191.

[19] Galor, O. and O. Moav (2004) From physical to human capital accumulation: inequality and the process of development. Review of Economic Studies 71, 1001-1026.

[20] Galor, O. and D.N. Weil (1996) The gender gap, fertility, and growth. American Economic Review 86, 374-387.

[21] Galor, O. and D.N. Weil (2000) Population, technology, and growth: from Malthusian stagnation to the Demographic Transition and beyond. American Economic Review 90, 806-828.

[22] Gollin, D. (2002) Getting income shares right. Journal of Political Economy 110, 458-474.

[23] Grandmont, J.M., P. Pintus and R. de Vilder (1998) Capital-labor substitution and competitive nonlinear endogenous business cycles. Journal of Economic Theory 80, 14-59.

[24] Guryan, J., E. Hurst and M. Kearney (2008) Parental education and parental time with children. Journal of Economic Perspectives 22, 23-46.

[25] Hall, R.E. (1988) Intertemporal substitution in consumption. Journal of Political Economy $96,339-357$.

[26] Hall, R.E. and C.I. Jones (1999) Why do some countries produce so much more output per worker than others? Quarterly Journal of Economics 114, 83-116.

[27] Havranek, T., R. Horvath, Z. Irsova and M. Rusnak (2015) Cross-country heterogeneity in intertemporal substitution. Journal of International Economics 96, 100-118. 
[28] Jones, C.I. (2004) The shape of production functions and the direction of technical change. NBER Working Paper No. 10457.

[29] Jones, L.E. and A. Schoonbroodt (2010) Complements versus substitutes and trends in fertility choice in dynastic models. International Economic Review 51, 671-699.

[30] Jones, L.E. and A. Schoonbroodt (2016) Baby busts and baby booms: the fertility response to shocks in dynastic models. Review of Economic Dynamics 22, 157-178.

[31] Jones, L.E. and M. Tertilt (2006) An economic history of fertility in the U.S.: 1826-1960. NBER Working Paper No. 12796.

[32] Jones, L.E., A. Schoonbroodt and M. Tertilt (2008) Fertility theories: can they explain the negative fertility-income relationship? NBER Working Paper No. 14266.

[33] Krueger, A.B. (1999) Measuring labor's share. American Economic Review 89, 45-51.

[34] Krugman, P. (1991) History versus expectations. Quarterly Journal of Economics 106, 651-667.

[35] Macunovich, D.J. (1998) Fertility and the hypothesis: an assessment of the literature. Journal of Population Economics 11, 53-111.

[36] Mankiw, N.G., D. Romer and D.N. Weil (1992) A contribution to the empirics of economic growth. Quarterly Journal of Economics 107, 407-437.

[37] Matsuyama, K. (1991) Increasing returns, industrialization, and indeterminacy of equilibrium. Quarterly Journal of Economics 106, 617-650.

[38] Palivos, T. (1995) Endogenous fertility, multiple growth paths, and economic convergence. Journal of Economic Dynamics 6 Control 19, 1489-1510. 
[39] Palivos, T., P. Wang and J. Zhang (1997) On the existence of balanced growth equilibrium. International Economic Review 38, 205-224.

[40] Wang, P., C.K. Yip and C.A. Scotese (1994) Fertility choice and economic growth: theory and evidence. Review of Economics and Statistics 76, 255-266. 


\section{Figures}

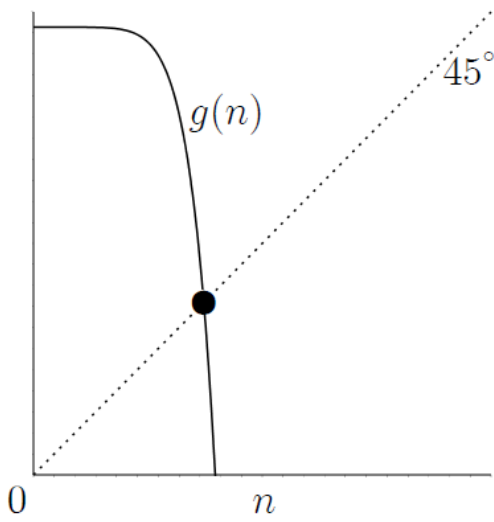

(a)

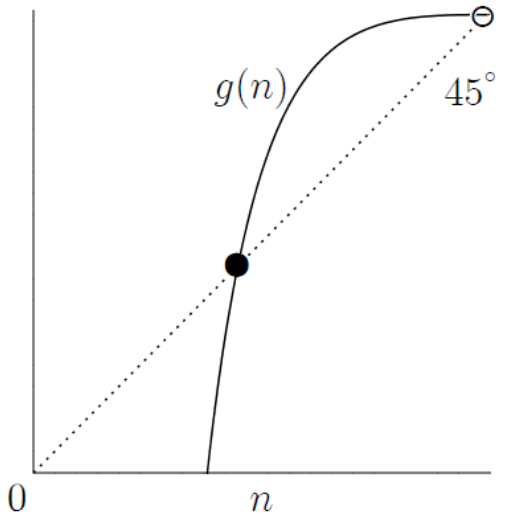

(c)

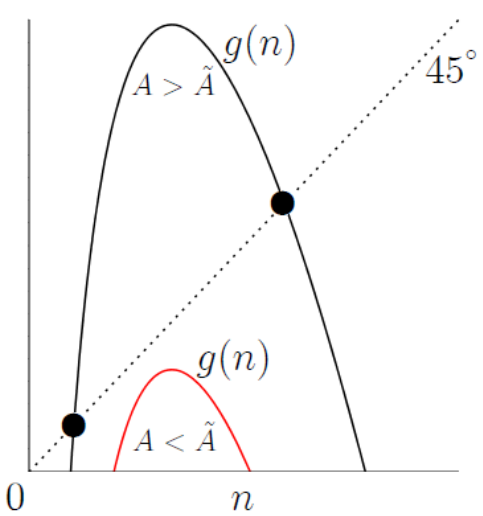

(b)

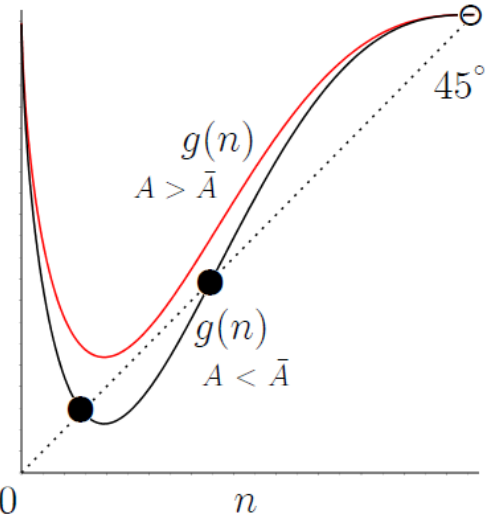

(d)

Figure 1. Geometry of existence and number of stationary states (denoted by the black point) of map $M$ as detailed in Proposition 3. (a) Case 1: $\sigma>1$ and $\gamma>\widetilde{\gamma}$. There is a unique stationary state. (b) Case 2: $\sigma>1$ and $\gamma<\widetilde{\gamma}$. If $A<\widetilde{A}$ there are no stationary states (red curve). If $A>\widetilde{A}$ there are two stationary states (black curve) (c) Case 3: $\sigma<1$ and $\gamma>\widetilde{\gamma}$. There is a unique stationary state. (d) Case 4: $\sigma<1$ and $\gamma<\widetilde{\gamma}$. If $A<\bar{A}$ there are two stationary states (black curve). If $A>\bar{A}$ there are no stationary states (red curve). In Case 3 and Case $4, n=2 / q$ (denoted by the empty circle) is not a stationary state of the map. However, it can play an important role for the dynamics of the model, as is shown later in this article. Case 4 illustrates the case of the existence of one inflection point. The case of the existence of two inflection points gives qualitatively identical results and then it is not reported. 


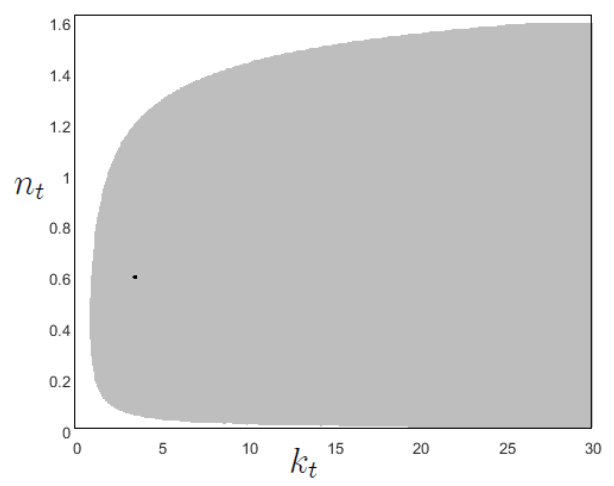

Figure 2. Parameter set: $\alpha=0.354, \sigma=4.01993, A=1.61, q=0.94$ and $\gamma=1.4485$. Local indeterminacy of the unique attractor of the map. The grey-coloured region is the basin of attraction of the attractor. The white area is the region of unfeasible trajectories.

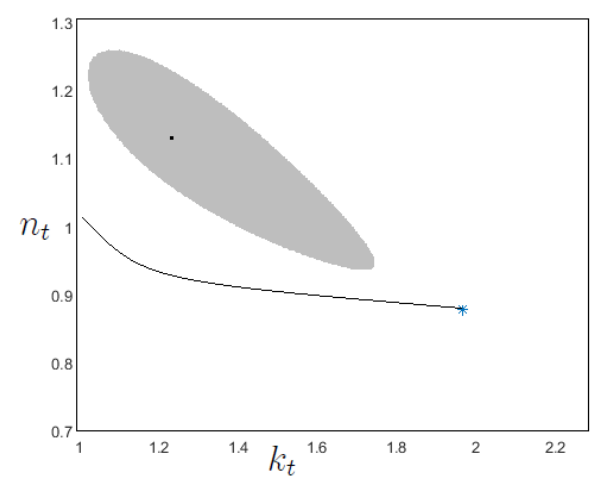

Figure 3. Parameter set: $\alpha=0.33, \sigma=4.7, q=0.53, A=1.545, \gamma=0.183$. Global indeterminacy. The grey-coloured region is the basin of attraction of $\left(k^{*}, n^{*}\right)$. The black line represents an approximation of a branch of the stable manifold of the saddle $\left(k^{* *}, n^{* *}\right)$ on which an economy converges towards the developed state. 


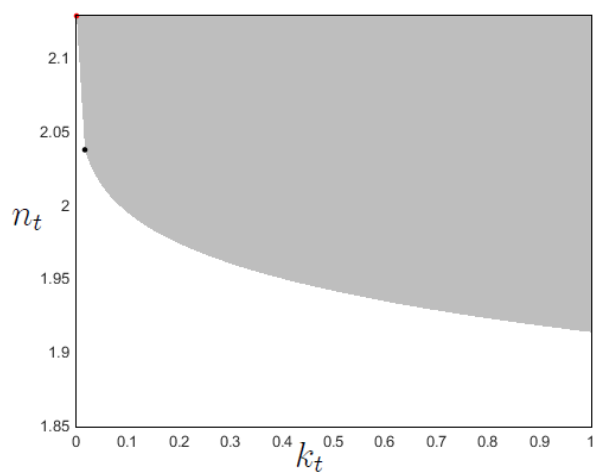

(a)

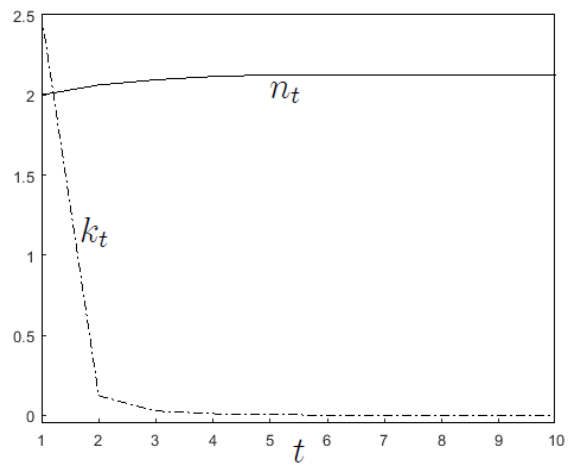

(b)

Figure 4. Parameter set: $\alpha=0.354, \sigma=1.3, A=1.07, q=0.94, \gamma=0.004$. (a) Basin of attraction (depicted in grey) of $(0,2 / q)$ (the red point) and the boundary of the basin that defines the stable manifold of the saddle (black) point $\left(k^{*}, n^{*}\right)$. (b) Time series of $n_{t}$ and $k_{t}$ of a trajectory approaching towards $(2 / q, 0)$.

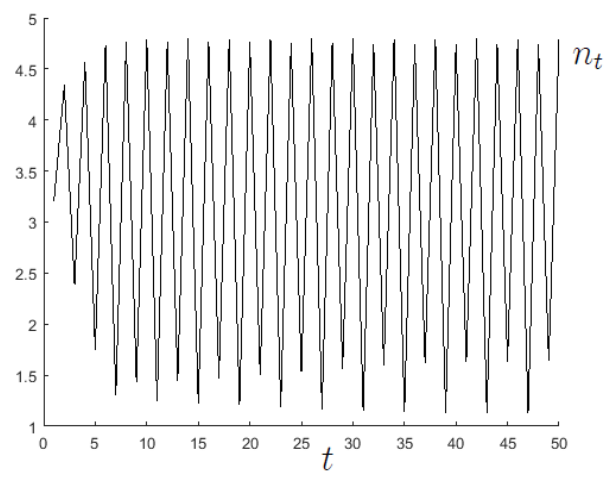

Figure 5. Parameter set: $\alpha=0.3, \sigma=1.956, A=1, q=0.3, \gamma=0.34$. Fertility fluctuations.

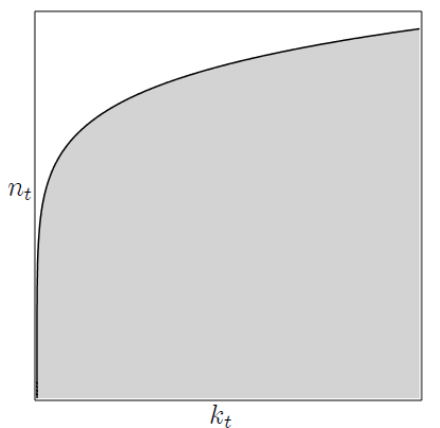

(a)

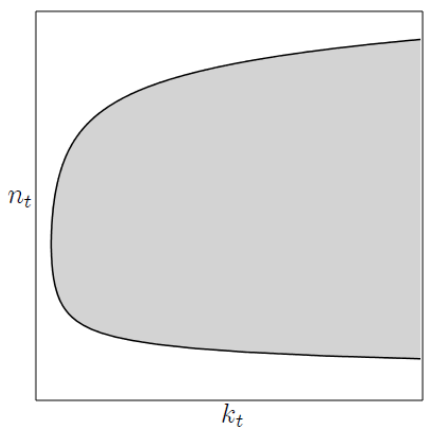

(b)

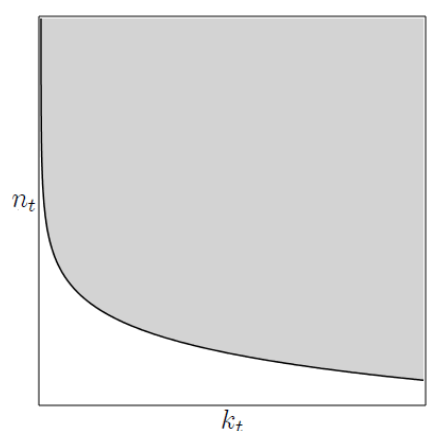

(c)

Figure A.1. Feasible region (grey-coloured) of map $M$ depending on the parameter setting. 